\title{
Clinical and Biochemical Evaluation of Subacute (De Ouervain's) Thyroiditis
}

\author{
M.Fariduddin', Nusrat-Sultana', M Badiuzzaman', ABMAbdullah, M.Abul Hasanat \\ ${ }^{1}$ Associate Professor of Endocrinology, Department of Medicine, BSMMU, ${ }^{2}$ Medical Officer of Endocrinology, Department of Medicine, \\ BSMMU, ${ }^{3}$ FCPS Course Student, Department of Medicine, BSMMU, ${ }^{4}$ Professor of Medicine, Department of Medicine, BSMMU, ${ }^{5}$ Associate \\ Professor of Endocrinology, Department of Medicine, BSMMU
}

\begin{abstract}
:
Background: Subacute thyroiditis is an important cause of thyrotoxicosis, often misdiagnosed as Graves' disease and given anti-thyroid drugs. Etiological diagnosis can yield proper guideline of management and a good result. Objectives: To see the clinical, biochemical and isotope profile of subacute thyroiditis. Methods: A total of 45 cases [36 females, 9 males; age (mean \pm SD): $33 \pm 4.7 \mathrm{yr}$ ] were recruited from Endocrine outpatient department of Bangabandhu Sheikh Mujib Medical University (BSMMU) to see thyroid hormones and isotope scan. Results: Most of the patients had a history of sore throat (84.4\%). All had painful thyroid gland with or without dysphagia, palpitation (93\%), fever (91\%), sweating and heat intolerance (80\%) and IBS like symptoms was 46\%. Among 36 females, 16 patients (44.4\%) presented with oligomenorrhea/amenorrhea. Clinically palpable thyromegaly was present in 33 cases (73\%), which was diffuse in 24 patients (73\%), resting tachycardia in 35(78\%) while anaemia in 23(51\%). A few cases (13/45) had raised blood pressure. Elevation of thyroid hormone was found in 44(97.7\%), but in most cases it was mild; little reduction of TSH (0.1-0.3mIU) was present in 43 (95\%). Characteristically, low radio-iodine uptake $(<5 \%)$ in 2 hours and 24 hours was found in $100 \%$ and thyroid scan revealed uniform uptake in all cases. ESR was raised in 100\% cases and leucocytosis was present in 9(20\%). Conclusion: It is concluded that subacute thyroiditis can be diagnosed on careful clinical examination. As it is a self limiting disease and does not usually need any anti-thyroid medication, subacute thyroiditis should be excluded in thyrotoxic patients before initiating anti-thyroid drugs.
\end{abstract}

Key words: Subacute thyroiditis, clinical and biochemical profile.

[BSMMU J 2010; 3(2): 82-85]

\section{Introduction:}

Subacute thyroiditis is an inflammatory disease of thyroid gland due to viral infection characterized by painful goitre, fever and symptoms of thyrotoxicosis such as palpitation, general malaise and sweating etc ${ }^{1}$. The diagnosis is often set clinically and is supported by the biochemical findings of mild to moderate hyperthyroidism and the suppressed ${ }^{131}$ I or ${ }^{99}$ Tc uptake by the thyroid gland ${ }^{2}$. The manifestations of thyrotoxicosis vary among patients. Younger patients tend to exhibit more sympathetic activations (e.g., anxiety, hyperactivity, tremor) while older patients have more cardiovascular symptoms (e.g., dyspnea, atrial fibrillation) and unexplained weight loss. The clinical manifestation of thyrotoxicosis does not always correlate with the extent of the biochemical abnormality. It is a destructive thyroiditis resulting in release of preformed thyroid hormone. Thyrotoxicosis from subacute thyroiditis is brief, usually lasting no longer than 6-8 weeks. It is a self-limited thyroid condition classically

Address for Correspondence: Dr. M.Fariduddin, Associate Professor of Endocrinology, Department of Medicine, Bangabandhu Sheikh Mujib Medical University (BSMMU), Dhaka, associated with a triphasic clinical course of hyperthyroidism, hypothyroidism, and return to normal thyroid function ${ }^{3}$. However, not infrequently it might be confused with sick euthyroid syndrome due to simulating pattern of hormone profile to some extent unless carefully correlated clinically and evaluated by radioisotope studies. A high degree of clinical suspicion is pivotal for diagnosis of subacute thyroiditis. On the other hand, though the gold standard for discrimination of subacute thyroiditis from other thyroid problems is the cytopathology, it is not always wise and feasible to study the cytopathology of a self-limiting disease of short duration ${ }^{4}$. Here we are reporting the clinical, biochemical and isotope profile of subacute thyroiditis recruited from Endocrine OPD and Institute of Nuclear Medicine (INM) of BSMMU.

\section{Methods:}

Subjects and protocol:

The study was carried out in the outpatient Department of Bangabandhu Sheikh Mujib Medical University. 45 subjects (9 male and 36 female) clinically suspected as subacute thyroiditis were recruited on consecutive basis from Endocrine OPD and INM of BSMMU. Diagnosis of 
subacute thyroiditis of every studied subject was confirmed by consultant endocrinologist. Age of the subjects was between 16 to 60 years. Characteristics of the studied subjects are shown in Table-1. The purpose of the study was clearly explained to each of the subjects and they were recruited only after they had given full consent. A thorough history and clinical examination was completed for each subject.

\section{Sample collection:}

Following the clinical examination, $4 \mathrm{ml}$ blood was collected using a disposable syringe in a plain glass tube. ESR and blood counts were done immediately. For hormone analysis, blood was kept at room temperature for clot formation. After centrifugation separated serum was collected in microcentrifuge tube to preserve below $-30^{\circ} \mathrm{C}$ until assay of hormones were done. All the blood tests were done in a unified way by the same institution and same personnel.

Analytic methods:

Thyroid hormones were assayed using commercial kits following ELISA methods in IMx ultra sensitive method (Abbott Park, Illinois, USA) or by RIA.

Radisotope scan and uptake:

Radioactive iodine uptake (RAIU) and isotope scan of thyroid gland was done in INM of BSMMU by the same personnel utilizing the same machine.

Data presentation:

All data are expressed in frequencies or percentages unless mentioned otherwise.

\section{Results:}

The present study encompassed 45 patients (Table-I) of subacute thyroiditis [age (mean $\pm \mathrm{SD}$ ): $33.3 \pm 4$.7 years] with various symptoms (Table-II) of the disease for a period of weeks to months. Almost all are taking iodized salt and 5 had family history of thyroid disorders while 4 had experienced some form of thyroid disorders in the past. All the patients had painful thyroid. Fever and palpitation were observed in more than $90 \%$ subjects followed by history of sore throat, constitutional symptoms, sweating and heat intolerance, weight loss, tremor (around 80\%). About 45\% patients had IBS like symptoms. Among of the females, 16 (44.5\%) had amenorrhea or oligomenorrhea. Palpable thyromegaly was observed in 33 (73.3\%) of which 25(55.5\%) were diffuse and 9 (27.2\%) were nodular. All patients had tender thyroid, 37 (82.2\%) had sweaty hands, 35 (77.7\%) had tachycardia, 30 (66.6\%) had tremor and 8 (17.7\%) showed hyper reflexia. Neutrophilic leucocytosis with lymphopenia was found among 9 subjects (Table-III).ESR was very high ( $>100 \mathrm{~mm})$ in 5 patients and moderately high (51-100 mm) in 34 patients while $<50 \mathrm{~mm}$ in 6 patients.

\section{Table-I}

Characteristics of subjects of the study $(n=45)$

\begin{tabular}{lc}
\hline Characteristics & Parameter \\
\hline Female / male & $36 / 9(4: 1)$ \\
Age (m \pm SD) & $33.3 \pm 4.7$ yrs \\
Duration of symptoms: & $<1$ month in 32 \\
& $>1$ month in 23 \\
Intake of iodized salt: & $98 \%$ \\
Family history of thyroid disorders: & 5 \\
History of previously thyroid disorders: & 4 \\
\hline
\end{tabular}

Findings are not mutually exclusive

Table-II

Clinical symptoms and signs

\begin{tabular}{llll}
\hline Symptoms & Number (\%) & Signs & Number(\%) \\
\hline Fever & $41(91.1)$ & Thyromegaly & $33(80)$ \\
Painful thyroid & $45(100)$ & Diffuse enlargement & $24(72.7)$ \\
Palpitation & $42(93.3)$ & Nodular enlargement & $9(27.3)$ \\
Sweating and Heat intolerance & $36(80)$ & Tenderness of thyroid gland & $45(100)$ \\
Tremor & $34(75.5)$ & Tachycardia & $35(77.7)$ \\
Weight Loss & $36(80)$ & Warm and sweaty hands & $37(82.2)$ \\
History of sore throat & $38(84.4)$ & Finger tremor & $30(66.6)$ \\
Constitutional symptoms & $38(84.4)$ & Hyper reflexia & $2(25)$ \\
IBS like symptoms & $21(46.6)$ & Anaemia & $5(21.7)$ \\
Amenorrheah Oligomenorrhea & $16(44.5)$ & Increased BP & $13(28.2)$ \\
\hline
\end{tabular}


Table-III

Blood counts and ESR ( $\mathrm{mm}$ in $1^{\text {st }}$ hour)

\begin{tabular}{lllll}
\hline Neutrophil Count & & Number & ESR(mm in $1^{\text {st }}$ hour, $\left.\mathrm{n}\right)$ & \\
\hline Normal TC & 36 & $<50$ & 6 \\
TC $>11000$ & Neutrophilia & 9 & $51-$ & 1 \\
& & & 75 & 2 \\
& Lymphopenia & 9 & $76-$ & 2 \\
& & 0 & 100 & 2 \\
TC $<4000$ & & $>100$ & 5 \\
\hline
\end{tabular}

Table-IV

T3, T4 and TSH levels in the studied subjects $(n=45)$

\begin{tabular}{lll}
\hline Variable & Frequency, n (\%) & \\
\hline Raised T3 & 3 \\
Raised FT3 & $40(88.88)$ & \\
Raised T4 & 3 & \\
Raised FT4 & $41(91.11)$ & $2(4.44)$ \\
Level of TSH & Normal (0.3-6mIU/L) & 38(84.44) \\
& Mild reduction(0.3-0.1mIU/L) & 5(11.11) \\
\hline
\end{tabular}

Groups are not mutually exclusive; T3 and T4 was not measured in all cases.

Elevation of FT3 (88.88\%) and FT4 (91.11\%) was observed in most of the subjects but magnitude of rise was not too high. In complement with this, TSH level was slightly reduced in about $84 \%$, but markedly reduced in $11 \%$ while normal in about 4\% (table-4). All the patients showed low RAIU and diffuse pattern of scan (Table-V).

Table-V

RAIU and isotope scan of the studied subjects

\begin{tabular}{llc}
\hline Variable & $\begin{array}{l}\text { Quality of Uptake } \\
\text { /Scan }\end{array}$ & $\begin{array}{c}\text { Frequency } \\
(\mathrm{n}, \%)\end{array}$ \\
\hline RAIU & $\leq 5 \%$ & $45(100)$ \\
\multirow{2}{*}{ Isotope scan } & Diffuse uptake & 0 \\
& Patchy uptake & $33(100)$ \\
\hline
\end{tabular}

Isotope scan was done in 33 patients

\section{Discussion:}

Subacute thyroiditis is a self- limited thyroid condition associated with a triphasic clinical course of hyperthyroidism, hypothyroidism, and return to normal function. It may be responsible for $15-20 \%$ of patients presenting with thyrotoxicosis and $10 \%$ of patients presenting with hypothyroidism ${ }^{3,4}$. Subacute thyroiditis can be diagnosed confidently on history and clinical examination, high erythrocyte sedimentation rate (ESR), leukocytes, abnormal thyroid function test (raised or normal T3 and T4 along with mild decreased TSH) and low RAIU by thyroid gland ${ }^{2}$.

In the present study we evaluated the clinical and biochemical profile of 45 patients of subacute thyroiditis who were confirmed of their clinical diagnosis by consultant endocrinologist. Almost all the patients showed features of mild thyrotoxicosis and thyroid hormones mimicked exactly the pattern of findings in classic subacute thyroiditis. However, unless careful and experienced enough, one may confuse the biochemical picture with that of Graves' thyrotoxicosis or sick euthyroid syndrome. TSH levels are usually suppressed to be of immeasurable levels $(<0.05 \mu \mathrm{IU} / \mathrm{ml})$ in Graves' thyrotoxicosis. In context to these facts, none of our patients were critically ill which is more frequent with sick euthyroid syndrome ${ }^{4}$. Moreover, neither fall of TSH nor rise of FT3 and FT4 were as high as to support Graves' thyrotoxicosis. In sick euthyroid syndrome T3 is rather frequently low or normal and there is no tender thyroid usually. Most of our patients had mild rise of FT3 and all of them had tender thyroid. Along with it, all patients showed very low uptake of radioactive iodine. Therefore, it is quite unlikely that our study has encompassed any sick euthyroid patients erroneously.

Antithyroid antibody titre is elevated temporarily in subacute thyroiditis. We did not measure it as it is not a diagnostic tool for diagnosis of subacute thyroiditis ${ }^{5}$. On the other hand, fine needle aspiration cytology (FNAC) or excision biopsy would have not been wise for diagnosis in a self-limiting disease of shorter duration unless it is really required for diagnostic decision making. It would also involve some cost for the patient. 


\section{Conclusion}

It is concluded that subacute thyroiditis can be diagnosed on careful clinical examination and is supported by low radioiodine uptake by thyroid. As it is a self limiting disease and does not usually need any anti-thyroid medication, subacute thyroiditis should be excluded in thyrotoxic patients before initiating anti-thyroid drugs.

\section{References:}

1. Pearce EN, Farwell AP, Braverman LE. Thyroiditis. N Engl J Med 2003;348: 2646-55.
2. Mittra ES, Niederkohr RD, Rodriguez C et-al. Uncommon causes of thyrotoxicosis.J Nucl Med 2008; 49: 265-278.

3. Lee SL, Ananthakrishnan S. Subacute Thyroiditis, eMedicine Endocrinology 2009 (Online).

4. Jameson JL, Weetman AP. Disorders of the thyroid gland. In: Kasper DL, Fauci AS, Longo DL, Braunwald E, Hauser SL, Jameson JL (eds.). Harrison's Principles of Internal medicine. $16^{\text {th }}$ edn, McGraw-Hill; New York, 2005: p 2117

5. Nishihara E, Ohye $\mathrm{H}$, Amino $\mathrm{N}$ et al. Clinical characteristics of 852 patients with subacute thyroiditis before treatment. Intern Med. 2008;47:725-9. 